\title{
Gender Prediction from Iris Recognition using Artificial Neural Network (ANN)
}

\author{
Sreya K C \\ Dept.of Electronics and communication \\ College of Engineering Thalassery \\ Kannur, India
}

\author{
Dr. Rini Jones S B \\ Dept.of Electronics and communication \\ College of Engineering Thalassery \\ Kannur, India
}

\begin{abstract}
Gender prediction from iris recognition techniques is widely used in human identification process namely biometric identification. Physical ageing is an important factor for biometrics. Now a days the biometric qualities such as face,hand geometry and fingerprints etc are used by the researchers to obtain the characteristics like hair colour, age ,gender, weight, ethnicity and so on. The iris recognition gives more accurate result comparing with other biometric verifications . This is a more verified technique for authentication while comparing to other biometric recognition systems, because he iris of two eyes of same individual are not comparative. The performance of iris recognition system enhanced the gender prediction from their iris images .This work pointed to enhance the gender prediction from eye images using artificial neural network .
\end{abstract}

Keywords-Gender prediction, iris, eye images, iris recognition

\section{INTRODUCTION}

Iris biometric is one of the most leading identification systems in the world. Many types of research are made under the iris biometric system.

Eye recognition emerges as one of the most useful tempers for bio-metrics recognition in the last few decennaries. The steps for iris recognition consists of mainly four parts: image acquisition, iris segmentation, feature extraction, and pattern matching. Because of its high recognition rate the iris recognition is one of the most meticulous biometric modality. several countries in the overall world have been enrolled in the iris recognition system for convenient use of passports, automated border crossing pass, and other identification processes to reduces the fake identity of the peoples.

In the case of human beings, we can easily recognize the gender. But there is some difficulty to be faced when using the computer vision technique while using the iris some features are used to identify the gender of the people

To Identify a person's gender[5] from an iris image such identification is related to forensics applications and security surveillance systems. a recognition system can detect iris of the peoples who have already been enrolled in the system. For persons who are not yet enrolled and so cannot be recognized, it may still be useful if the system could yield some basic demographic information for the person.

Gender prediction is essential and critical for many applications in the commercial domains. It is simple for an independent to recognize the gender however it is exceptionally hard for the machines. Gender recognition is applicable in various fields, for example, security frameworks, forensic sciences, etc. Various strategies were utilized in previous years to characterize gender utilizing facial images although only a few types of research have used the features of iris images to foresee the gender. The biometric authentication system dependent on iris images is commonly viewed as more solid than other frameworks. Numerous researchers have used facial images for the recognition of gender. Iris images have been utilized for recognition purposes, but there exist some references revealing the use of iris images for identification of human attributes.

Ongoing through the paper is to predict the gender using the eye image data. The image is normalized after normalization the prediction can be done by using ANN.The prediction the gender by an assumption.

The discuss as follow in section II, III, IV . In section II will discuss the block diagram .Section III will be the experimental result also section will conclude the paper.

\section{BLOCK DIAGRAM}

The gender prediction from iris recognition can be done by mainly seven stages. These stages are:
A. Image acquisition
B. Image localization
C. Image segmentation
D. Iris normalization
E. feature encoding
F. ANN(Training)
G. Gender prediction

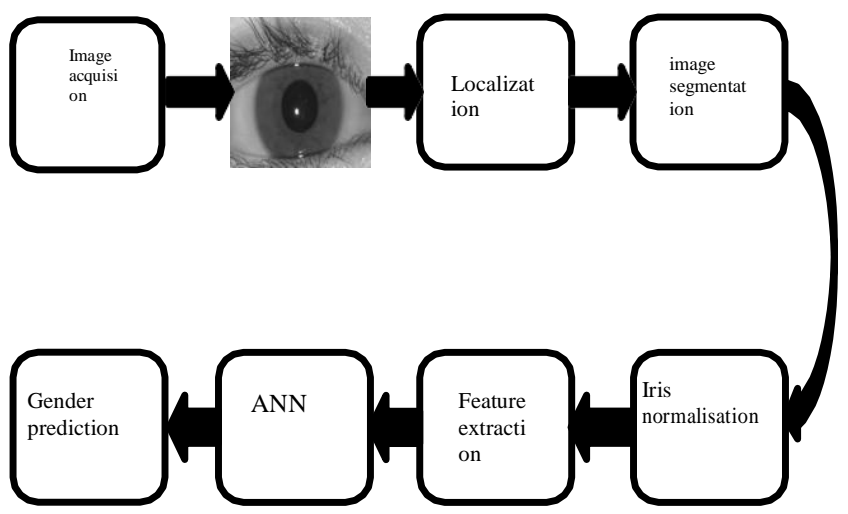

Fig1: block diagram of gender detection 


\section{A. Image Acquisition}

The iris image should be image quality abundant in iris texture. The eye image is collected from IT Delhi data set and also captured iris image using iris scanners.

\section{B. Localization}

Iris Recognition is the most accurate biometric identification system. The image processing using concepts of neural networks the Iris Recognition of a person is identified by the iris which is the part of the eye . To identify a person's gender with high efficiency and high accuracy by analyzing the random patters visible within the iris. It can be implemented by using a modified Canny edge detection algorithm.

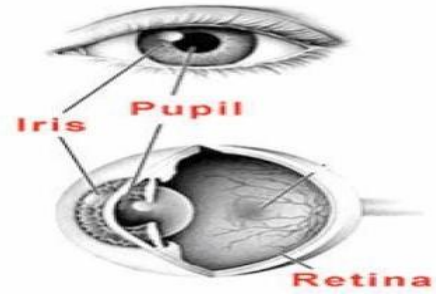

Fig2: the human eye

\section{Image Segmentation}

In the primary step take the digital images then the iris recognition is to isolate the original iris region. The iris region, shown in the above figure2, can be approximated by two circles, one for the iris/sclera boundary and another, interior to the first circle, for the iris/pupil boundary. After completing the segmentation process, the segmentation depends on the imaging quality of the eye images.The inner boundary and outer boundary of the iris are placed . Canny edge detection used for finding the edge of the eye image.

The algorithm done is done 5 steps:

1. Smoothing: blurring and filtering is used to remove the noise of the eye image.Gaussian blur will reduce the high frequency components from the image; thus Gaussian blur can be considered as a low pass filter.

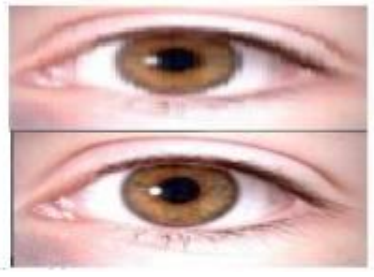

Fig 3. Blurred and filtered eye image

2. Non-maximum suppression: The edges should be marked when a part of the image to be processed is circular and nonlinear boundary region matching a similar shape is taken out for only local maxima.It remove the pixels that are not considered to be the part of an edge

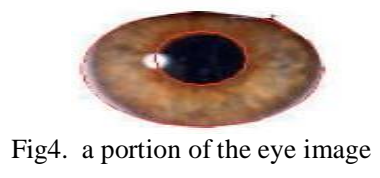

3.Thresholding : Detect the Potential edges of the eye image by using the threshold.There are two types of thresholds they are upper and lower thresholds. If the intensity gradient of a pixel is higher than the upper threshold, the pixel will be accepted as an edge. If it is below the lower threshold, then it will be rejected. If it is in between these two thresholds, the pixel will be accepted only if it's adjacent pixel has intensity gradient above the upper threshold. Thresholding is used to convert iris image to binary image and then morphological operations such as opening and closing is applied on the binary image to get the pupillary region.

4.Canny Edge Detection: .It reduce the data to be processed based on the intensity gradient of the image.Thus the unwanted portions like sclera, pupil etc are removed.

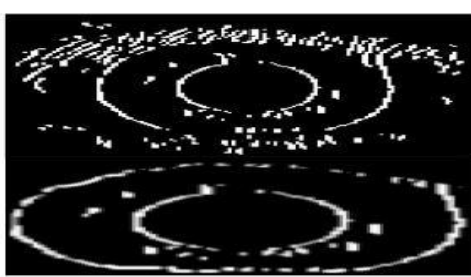

Fig 5.result of Canny edge image

5. Hough Transform : The Hough transform is a standard computer vision algorithm that can be used to designate the parameters of simple geometric objects, such as circles and lines present in an image. The center and radiuscoordinates of the pupil and iris regions can be worn to deduce by using circular Hough transform transform(CHT). CHT is a feature extraction technique used for identifying the circles. The function of this method is to find circles from imperfect image inputs.

\section{Normalization of Image}

After the iris segmentation, the upcoming step is to normalize this image, to enable the iris code and their comparisons. optical size of the iris, pupil position in the iris, and orientation of the iris are different in different peoples, it is required to normalize the iris image, so that the representation is common to all, with similar dimensions. To unwrapping the iris and transform it changes into its polar equivalent has done by using the normalization process. It can be done by using Daugman's Rubber sheet model . while considering the reference point will be the center of the pupil in eye image.

\section{E. Feature Extraction}

normalized image are transformed to gray image and then to binary image. Centroid, area and bounding box from the binary images of the normalized eye images are extracted. Mean, Variance, and standard deviation of the normalized eye images are also calculated. 


\section{F. Artificial Neural Network}

Using artificial neural networks to calculate, the desired output for any given input. Using Feed-forward networks (networks that have no feedback, ). It has two parts: Training and Testing

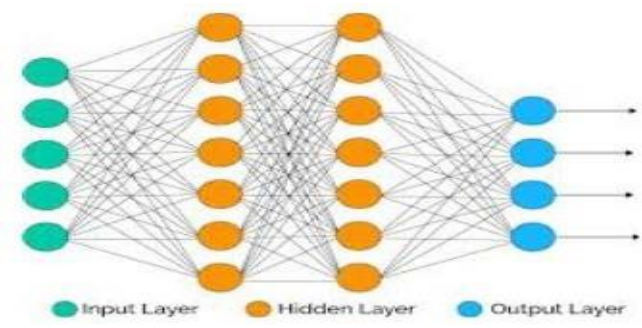

Fig 6:architecture of ANN

The input layer is the first layer which indicates the input and the last layer is the output layer that represents the output of the network. The remaining layers are called hidden layers. These layers are having interconnection nodes that are associated with weights. For a neural net with supervised training, the training is carried out by providing a sequence of Training data each with an associated target output. Finally, in the testing phase, the normalized eye image which is given as input is transformed to grayscale and then to binary, and the features are extracted. Then take the trained data, it is identified that, whether the testing iris belongs to male or female.

Need training data set to teach the neural network. The training data set consists of inputs assigned with the corresponding desired output. the first step is to start the teaching process with a large value of the parameter.The weights coefficients are being established the parameter is being decreased continuously . Support-vector machines (SVMs,also support vector networks)are supervised learning models with associated learning algorithms that analyze data used for classification and regression analysis.

\section{EXPERIMENTAL RESULT}

The Python and Matlab 2017b is used to do the feature extraction and the training steps. data set of iris images were collected from IIT Delhi .Fig7 image from the data set

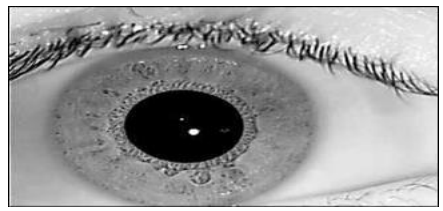

Fig. 7. An iris image from data set
For iris localization to detect the edges by applying the Canny edge detection, followed by Gaussian blurring to remove noises, finally Circular Hough transform is applied on the Gaussian blurred image to detect the area of limbic boundary. Fig. 8shows the result of Canny edge detection and Fig. 9 shows the Gaussian blurred image. Using the values of center and radius and limbic boundary is plotted on the iris image as in Fig. 10. Iris image is cropped in such a way that the limbic boundary is fit in that image as shown in Fig. 11

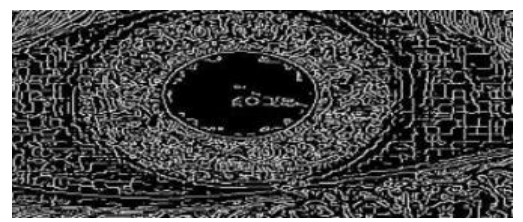

Fig.8 Canny edge detected image

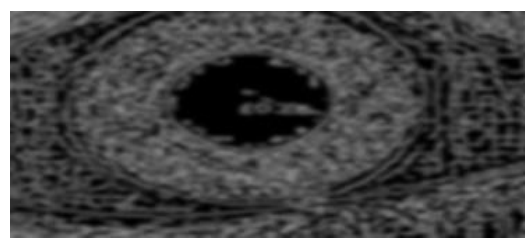

FIG. 9 Gaussian Blurred Image

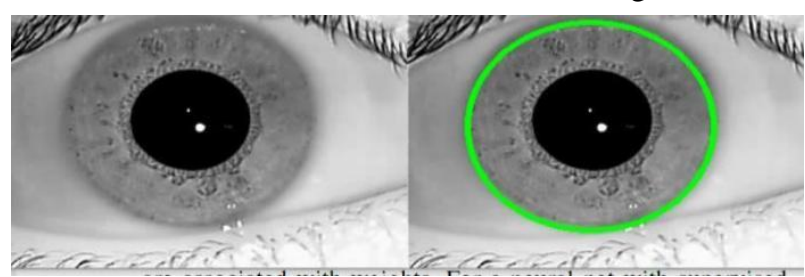

Fig10: Circular Hough Transformed Image

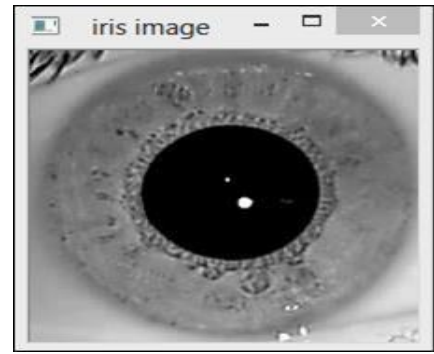

Figure11: iris image

To obtain the pupil region, the cropped image and binarized using thresholding. The morphological closing, eroding, and an opening is applied on the image to get the pupillary region as

Fig.12. 


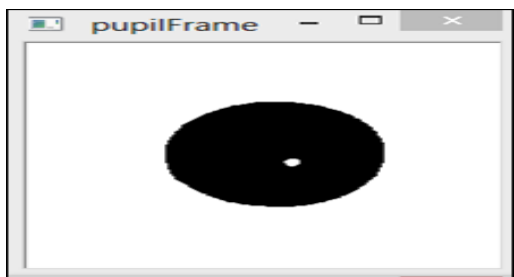

Fig. 12. Image after morphological operations

To get the pupillary region alone, a function is defined to return the rows and columns of the black region that is pupil. Thus the radius and center of the pupillary boundary are easilyfound from these rows and columns . Using thesecenters and radius obtained, the limbic and pupillary boundaries are drawn on the cropped iris image as shown in Fig. 13

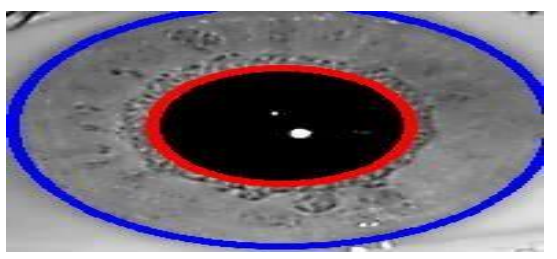

Fig13 .limbic and Pupillary boundaries detected

The width of the iris region can be obtained by subtracting the pupillary radius from the limbic radius. Daughman's Rubber Sheet model was applied to eye image to get a normalized image as shown in Fig. 14. Thus normalized images of 40 eye images were generated to create a database for training purposes. After the localization and segmentation steps, the remaining steps were done using Matlab software.

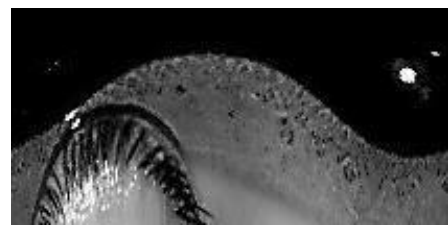

Fig14.normalized image

In feature extraction, Features are extracted after converting normalized images to gray, and then to binary .40 normalized eye images were used to extract features such as centroid, area, and bounding box. Out of 40 normalized images, 20 images belongs to male and 20 to female. From featurescalculated, it is noted that the area and bounding box calculated from the normalized images of males are smaller than the values obtained from the normalized images of females. Thus the feature includes $\mathrm{x}$ and $\mathrm{y}$ coordinates of the centroid, area of the region, and the bounding box values (x, y, w (width), $\mathrm{h}$ (height)). Therefore there are seven features are used for train the neural network.

Mean, Variance, and standard deviation of the normalized eye images were also calculated to get additional features to train the network. The mean value is 0.5 , Variance is 0.0876 , the Standard deviation is 0.2957 are same for all the images. Since those values are the same for all the images, these values can be used as a feature to train the network. A target database is created in such a way that 1 is denoted to represent male normalized eye image and 2 to represent female normalized eye image.

Giving the eye feature data set as input and target gender dataset as output of the neural network is created as shown in Fig. 15

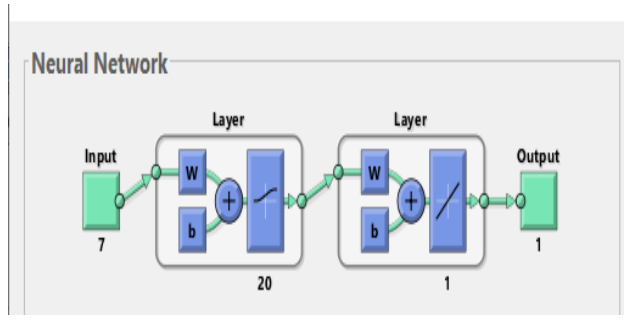

Fig. 15. Neural Network

It is a feed-forward neural network with 20 neurons, 8000 epoch, and 0.01 learning rate. Seven features are the input to the neural network to get a single output. Then training function is applied to train the network and net function is used to obtain the output. In the testing phase, 20 normalized eye images were used, in which 11 belongs to females and 9 belongs to male.

Here the normalized eye image which is given as input is converted to gray and then to binary and the features are extracted. Here thelabeled data is loaded. Then according to trained data and the value of the features of the testing image, gender label is detected as 1 if it is an eye image of males and 2 if it is an eye image of a female.

Fig. 16 shows the example of the values of features calculated when a testing image is given as input. Fig. 17 shows the result calculated according to the obtained features and trained data. Here this testing image is identified as the eye image of a male. If the testing image belongs to female an example of feature values obtained are shown in Fig. 18 and the gender recognition result is as shown in Fig. 19

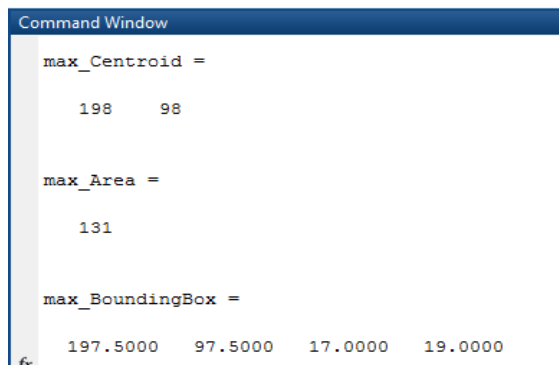

Fig.16 Features Calculated(Male)

$$
\begin{aligned}
& \text { Detected_Gender_Label }= \\
& 1 \\
& \text { male }
\end{aligned}
$$

Fig 17 Predicted the Gender as Male 


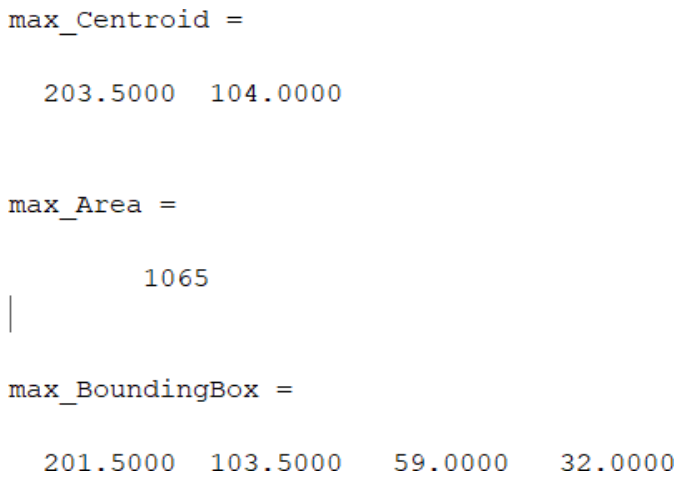

Fig. 18 F eatures Calculated(Female)

Detected_Gender_Label =

2

Female

Fig 19 Predicted The Gender as Female

\section{CONCLUSION}

Identify a person's gender from an iris image that identification is used for security surveillance systems and forensics applications. The neural network which can easily predict the gender from iris using certain training process . The accuracy will depend on processing step .More better processing will give better accuracy. The gender classification features detect the area, center,bounding box of the normalized iris image comparing the area and bounding box of the normalized irisimages the values of males are smaller than females.Also main observation is that the area of female iris region are larger than that of male iris region.

\section{REFERENCES}

[1] J. Daugman, Biometric personal identification system based on iris analysis. US Patent 5291560, 1 Mar 1994

[2] Fenker S. and Bowyer K., "Analysis of Templat Aging in Iris Biometrics," in Proceedings IEEE Computer Society Conference on Computer Vision and Pattern Recognition Workshops, Providence, pp. 45-51, 2012.

[3] M. Erbilek and M. Fairhurst, "Framework for managing ageing effects in signature biometrics,"Biometrics, IET, vol. 1, pp. 136-147, 2012

[4] N. Cristianini. and J. Shawe-Taylor., "An introduction to support vector machines and other kernel-based learning methods,"Robotica, vol. 18, pp. 687-689, 2000.

[5] Ortiz E., Bowyer K., and Flynn P., "A Linear Regression Analysis of the Effects of AgeRelated Pupil Dilation Change in IrisBiometrics," in Proceedings of the $6^{\text {th }}$ International Conference on Biometrics: Theory, Applications and Systems, Arlington, pp. 1-6, 2013.

[6] K.W. Bowyer, S. Lagree and S. Fenker, "Human versus biometric detectionof similiarty in left and right irises", IEEE International Carnahan Confernce on Security, October 2010.

[7] S. Lagree and K.W. Bowyer, "Ethnicity prediction based on iris texture features", 22nd Midwest Artificial Intellignece andCognitive Science Conference (MAICS), April 2010.N.

[8] D. Kalka, J. Y. Zuo, N. A. Schmid, "Estimating and fusing qualityfactors for iris biometric images", IEEE Transactions on SystemsMan and Cybernetics:Part A Sys-tems and Humans, vol. 40, pp. 509-524, May 2010.
[9] J. Canny: A Computational Approach to Edge Detection. IEEE Transaction on Pattern Analysis and Machine Intelligence Vol. 8 (1986) 679-714

[10] W.W. Boles, B. Boashah: A Human Identification Technique Using Images of the Iris and Wavelet Transform. IEEE Transaction on Signal Processing Vol. 46 (1998) 1185-1188 